\section{Microscale Cantilever Beam Resonators "Weigh" Individual Virus Particles}

Nanotechnology researchers are pursuing the fabrication of a lab-on-a-chip device to detect biochemical entities such as airborne virus particles. As reported in the March 8 issue of Applied Physics Letters, A. Gupta and co-workers at Purdue University have taken a mechanical approach and fabricated an array of microscale resonant cantilever beams, which act as mass detectors. The frequency of oscillation of each cantilever is measured using a microscope scanning laser
Doppler vibrometer under ambient conditions. The cantilevers are small enough that they do not require an external source, but are driven by thermal and ambient fluctuations. The researchers dispersed a solution of vaccinia virus particles (vaccinia is a member of the Poxviridae family and forms the basis for the smallpox vaccine) in deionized water over the cantilever array and allowed the particles to incubate for $30 \mathrm{~min}$ before drying the array. They showed that the attachment of a single vaccinia virus particle, with a mass of about $10 \mathrm{fg}$, shifts a $1.27 \mathrm{MHz}$ resonant frequency by $5 \%$. The researchers have demonstrated that the shift in the natural frequency is proportional to the effective number of virus particles on the beam, as expected from a mechanical analysis, by imaging the cantilevers with a scanning electron microscope and counting the number of virus particles attached.

The scientists started with $p$-type siliconon-insulator wafers and photolithographically patterned and etched the arrays of 5 - $\mu \mathrm{m}$-long cantilevers in the silicon. The cantilevers are 1-2 $\mu \mathrm{m}$ wide and 20-30 $\mathrm{nm}$ thick. After protecting the cantilevers with an oxide etch-stop layer, the research team used a xenon difluoride

\section{High-Speed Silicon Optical Modulator Developed}

Silicon, the material of choice for electronics, has not seen wide consideration as an optical material for photonics. A key limitation has been the relatively low speed of silicon optical modulators compared with other materials such as III-V compounds and lithium niobate. Researchers from Intel, led by A. Liu, have now reported the fabrication of an all-Si optical modulator with a modulation bandwidth exceeding $1 \mathrm{GHz}$. This represents a nearly two orders of magnitude improvement over previous $\mathrm{Si}$ devices, which have modulation frequencies in the range of $\sim 20 \mathrm{MHz}$. Liu and co-workers described their approach, based on a metal oxide semiconductor (MOS) capacitor structure embedded in a Si waveguide, in the February 12 issue of Nature.

In this device, the modulation of light for encoding data as changes in intensity is achieved by modulating the refractive index. In $\mathrm{Si}$, this is achieved by the free-carrier dispersion effect; the introduction of free carriers results in absorption and also a change in the refractive index. The researchers used a MOS capacitor phase shifter for charge-density modulation that induces a phase shift in the optical mode. Their device consists of an $n$-type Si slab and a $p$-type doped polysilicon rib with a gate oxide layer sandwiched in between. The poly-Si was formed by chemical vapor deposition of amorphous $\mathrm{Si}$ and subsequent annealing under conditions that minimize

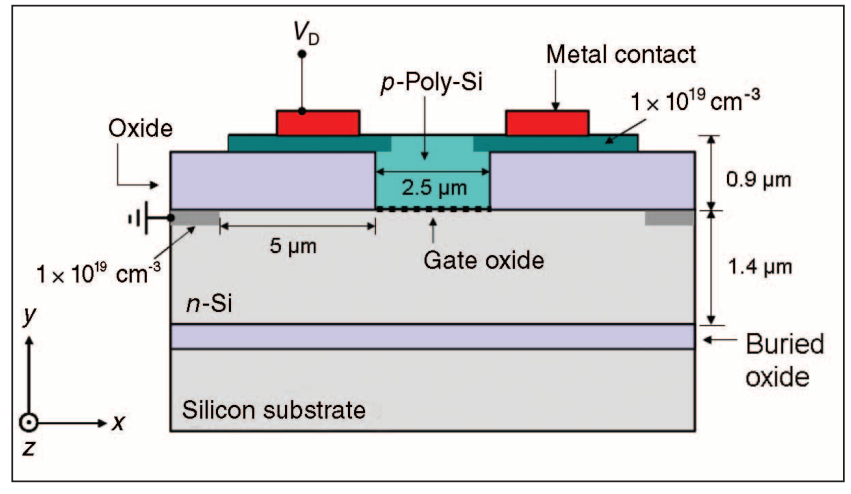

Figure. Schematic diagram shows the cross-sectional view of a metal oxide semiconductor capacitor waveguide phase shifter using silicon-on-insulator technology, where $\mathrm{V}_{D}$ is drive voltage and $1 \times 10^{19} \mathrm{~cm}^{-3}$ is the doping density. (Nature 427 [2004] p. 616.) grain-boundary formation. Aluminum contacts were deposited on the sides of the rib waveguide (see Figure). The modulators were fabricated in an existing Intel complementary MOS (CMOS) production facility, demonstrating the use of existing infrastructures for fabricating these and similar devices and indicating the potential ease of integration of optoelectronic components into CMOS microelectronics. Modeling and testing confirmed that this was a single-mode optical device at a wavelength of $\sim 1.55 \mu \mathrm{m}$, which is widely used in optical-fiber communications.

To test the modulators, an asymmetrical Mach-Zehnder interferometer (MZI) was fabricated, with two identical MOS capacitor phase shifters in two arms of the MZI, to convert the phase modulation into an intensity modulation. The MZI had an optical path length difference of $16.7 \mu \mathrm{m}$ between the two arms; Y junctions allowed for the splitting and recombination of the optical beams. The MZI had an on-chip insertion loss of $6.7 \mathrm{~dB}$, a phase shifter length $L$ of $1 \mathrm{~cm}$, a $V_{\pi} L$ of $8 \mathrm{~V} \mathrm{~cm}$ (where $V_{\pi}$ is the voltage required to produce a $\pi$ phase shift), and an extinction ratio of $>16 \mathrm{~dB}$. Results demonstrated that the MZI modulator showed a roll-off frequency of $>1 \mathrm{GHz}$ by the induced phase shift. This modulation frequency is two orders of magnitude higher than that observed for traditional current-injection-based diode devices. The data transmission performance of the MZI modulator was tested using a digital pulse drive voltage. A pseudo-random electrical data input was used, and the corresponding MZI optical output was measured. The output optical signal faithfully reproduced the $1 \mathrm{Gbit} / \mathrm{s}$ electrical data stream.

The researchers said that the modulator performance could be further improved by replacing the polysilicon with singlecrystal $\mathrm{Si}$, which could reduce the on-chip insertion loss by $\sim 5 \mathrm{~dB}$. Work on this is currently under way. Reducing the waveguide dimensions and making the gate oxide thinner would also reduce the optical loss as well as device size. The implications of this study could be far-reaching. It has clearly catapulted $\mathrm{Si}$ into the research spotlight as a strong candidate material for photonic applications. In particular, since a strong fabrication infrastructure already exists for Si processing, it may be much easier to quickly incorporate Si into the photonics industry and rapidly achieve economies of scale similar to those found in the electronics industry. A low-cost silicon optical superchip could become a reality very soon.

Gopal RaO 
vapor-phase etch to take away the underlying silicon, and then removed the oxide shell with a buffered hydrofluoric acid etch, which releases the silicon levers.

Once antibody-based recognition is implemented to form an "immuno chip," these ultra-sensitive nanomechanical oscillators may prove to be rapid, economical biosensors, said the researchers.

RICHARD LOUIE

\section{Damage Threshold of Extreme- Ultraviolet Multilayer Mirrors Measured}

The rapid advance of coherent extremeultraviolet (EUV) sources, such as freeelectron lasers, requires the development of new optical materials. Among them are high-reflectivity mirrors, which are usually prepared by producing multilayer coatings of $\mathrm{Mo} / \mathrm{Si}, \mathrm{W} / \mathrm{C}, \mathrm{W} / \mathrm{Si}$, and, more recently, Sc/Si. However, damage to the mirrors when exposed to high peak powers of EUV light presents a problem because the peak power and fluence of EUV sources have recently increased significantly. For example, the radiation fluence of a capillary-discharge neon-like Ar laser operating at $46.9 \mathrm{~nm}$ can exceed $1 \mathrm{~J} / \mathrm{cm}^{2}$. In the March 15 issue of Optics Letters, M. Grisham of Colorado State University, Yu.P. Pershyn of the National Technical University in the Ukraine, A.V. Vinogradov of the P.N. Lebedev Physical Institute in Moscow, and their colleagues have studied the damage of Sc/Si multilayer mirrors exposed to intense EUV laser pulses.

The researchers prepared the multilayer mirrors by dc magnetron sputtering. The multilayers consisted of several periods of Sc/Si pairs, each with a thickness of $26.7 \mathrm{~nm}$ and a ratio of layer thickness of $\mathrm{H}(\mathrm{Sc}) / \mathrm{H}(\mathrm{Si})$ of 0.7. A 5-nm-thick Si protective layer capped the multilayers. Mirrors were fabricated on substrates of silicon and borosilicate glass. The irradiation was conducted by focusing the output of a tabletop capillary-discharge neon-like Ar laser emitting $1.2 \mathrm{~ns}$ pulses at a wavelength of $46.9 \mathrm{~nm}$. Scanning and transmission electron microscopy (SEM and TEM) and small-angle $\mathrm{x}$-ray diffraction (XRD) techniques were used to analyze the multilayers after irradiation. For some of the tests, the sample was moved after each laser shot, so that the site was hit by only a single pulse. In other tests, the sample was moved relative to the laser beam, so that a large area was damaged to permit small-angle XRD measurements to be performed. The results of the SEM, crosssectional TEM, and small-angle XRD measurements show that the damage mechanism is of a thermal nature, with evidence of melting and even boiling of the material at high laser fluence. A damage threshold of $0.08 \mathrm{~J} / \mathrm{cm}^{2}$ was measured, comparable to the $0.7 \mathrm{~J} / \mathrm{cm}^{2}$ found necessary to damage a bare Si substrate. This value is similar to the thresholds found in $\mathrm{Mo} / \mathrm{Si}, \mathrm{W} / \mathrm{C}$, and W/Si coatings measured at much shorter wavelengths.

ROSALIA SERNA

\section{Single ZnS Nanoribbons Lase under Optical Pumping}

Nanowires have exciting applications as building blocks of nanodevices. However, they are difficult to study due to their small size. Nanoribbons are easier to study because their larger size allows easier access, manipulation, and processing. In the February 16 issue of Applied Physics Letters, S.T. Lee from the City University of Hong Kong, X.M. Meng from the Technical Institute of Physics and Chemistry in Beijing, and co-workers report the fabrication of ZnS nanoribbons that were then studied using transmission electron

\section{MRS Online}

\section{E-Mail Alerts}

Sign up for any of these FREE services today and let the Materials Research Society bring materials information to you!

- eMatters

- MRS Table of Contents Alert

- Just Published! Book Alert

- MRS Meetings Alert

- MRS Meeting Scene

- MRS Public Affairs Alert

- Women in MS\&E

\section{For more information, go to... www.mrs.org/alerts/}

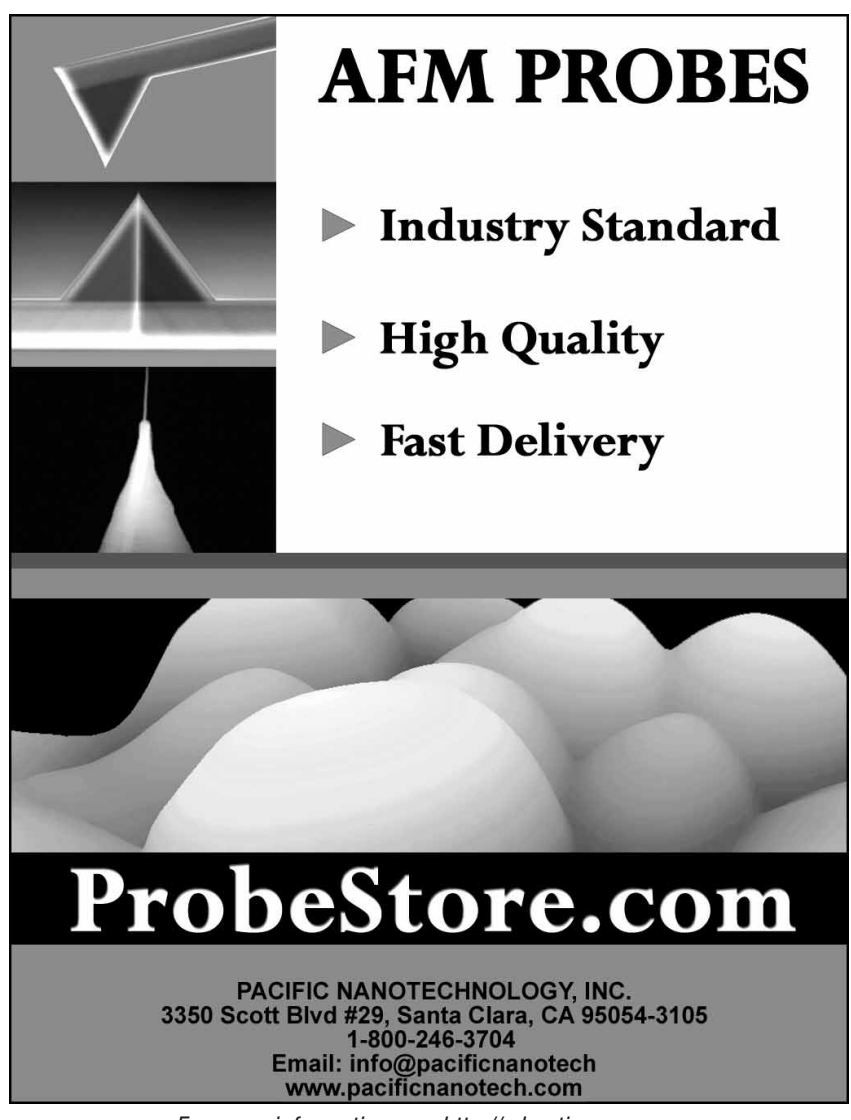

For more information, see http://advertisers.mrs.org 